\title{
Stimulation de la moelle épinière : solution de rechange non opioïde à la gestion de la douleur chronique
}

\author{
Aaron Hong MD MSc, Vishal Varshney MD, Gregory MT Hare MD PhD, C. David Mazer MD
}

- Citation : CMAJ 2020 October 19;192:E1264-7. doi : 10.1503/cmaj.200229-f

Voir la version anglaise de l'article ici : www.cmaj.ca/lookup/doi/10.1503/cmaj.200229

$\mathbf{L}$ a douleur chronique affecte 1 Canadien sur 5 et est associée à un fardeau socio-économique considérable ${ }^{1}$. Même si les opioïdes ont été la pierre angulaire du traitement, leur popularité est en baisse en raison des crises liées à la toxicomanie, au mésusage, à l'épuisement de l'effet et à la dépendance ${ }^{1,2}$. Des solutions de rechange ont donc été conçues, comme la thérapie cognitivo-comportementale, la réadaptation physique, la pharmacologie sans opiacés et les thérapies intégratives ${ }^{1,3}$.

Lorsque les traitements classiques entraînent des effets indésirables inacceptables ou ne soulagent pas suffisamment la douleur, la stimulation de la moelle épinière (neuromodulation) peut constituer une option de secours, seule ou avec d'autres modalités ${ }^{3,4}$.

La neuromodulation, définie comme une altération de l'activité nerveuse au moyen de stimuli ciblés, a fait son apparition en $1967^{2,3,5}$. Elle se fonde sur un principe de stimulation électrique de la colonne dorsale de la moelle épinière, dans le but de masquer les signaux douleureux². La technologie a depuis évolué et c'est un neurochirurgien canadien qui a aidé à en démontrer l'efficacité et la rentabilité pour le traitement de la douleur chronique ${ }^{6,7}$.

L'utilisation des stimulateurs de la moelle épinière a augmenté dans le monde et la Food and Drug Administration des États-Unis estime à 50000 le nombre de dispositifs implantés annuellement aux États-Unis (150 cas/million de population; www .fda.gov/medical-devices/letters-health-care-providers/conduct -trial-stimulation-period-implanting-spinal-cord-stimulator-scs -letter-health-care-providers). Toutefois, au Canada, son adoption a été substantiellement moindre, soit 172 implantations en Ontario en 2018 (12 cas/million de population) $)^{3}$. Cette différence a probablement à voir, du moins en partie, avec la méconnaissance de cette modalité et le coût initial élevé des dispositifs $(20000 \$-30000 \$)^{3}$.

\section{Qu'est-ce que la stimulation de la moelle épinière?}

La stimulation de la moelle épinière se fait au moyen d'électrodes posées sur l'espace épidural le long de la colonne dorsale et connectées par voie sous-cutanée à un générateur

\section{POINTS CLÉS}

- La stimulation de la moelle épinière masque les signaux douloureux au moyen d'un générateur d'impulsions électriques transcutané implantable.

- La stimulation de la moelle épinière est sécuritaire, efficace et économique pour le traitement de la douleur chronique due à des maladies douloureuses neuropathiques, y compris le syndrome d'échec de la chirurgie du dos, le syndrome régional douloureux complexe et les neuropathies périphériques chroniques.

- Les nouvelles technologies de stimulation de la moelle épinière ont des indications cliniques plus diversifiées, notamment la douleur viscérale et ischémique, et offrent un potentiel d'amélioration de leur efficacité.

- En améliorant les connaissances sur le traitement par stimulation de la moelle épinière et son accessibilité, on permettra à plus de Canadiens de profiter du soulagement d'une douleur chronique rebelle et on réduira la consommation d'opioïdes.

d'impulsions implantable. Ces générateurs sont munis d'une pile et d'un microprocesseur (comme celui d'un stimulateur cardiaque); ils sont insérés dans une pochette sous la peau au niveau de la paroi abdominale, du flanc ou du haut de la fesse), et programmés par voie transcutanée avec contrôle à distance par le médecin ou le patient. La durée de vie des générateurs d'impulsions implantables est de 5-10 ans selon leur rechargeabilité, et ils sont remplaçables. On demande aux patients de ne pas exercer de torsion, de flexion ou d'étirement excessifs pendant une période de 6 à 8 semaines après l'implantation. Après cette période, les patients peuvent reprendre la plupart des leurs activités physiques.

Les générateurs émettent en continu des impulsions électriques de basse fréquence (stimulation tonique) pour masquer les signaux douloureux qui voyagent le long de la moelle épinière et les remplacer par des paresthésies non douloureuses ${ }^{8}$. La théorie dite du " portillon ", de Wall et Melzack, est la plus généralement acceptée parmi les mécanismes proposés pour 


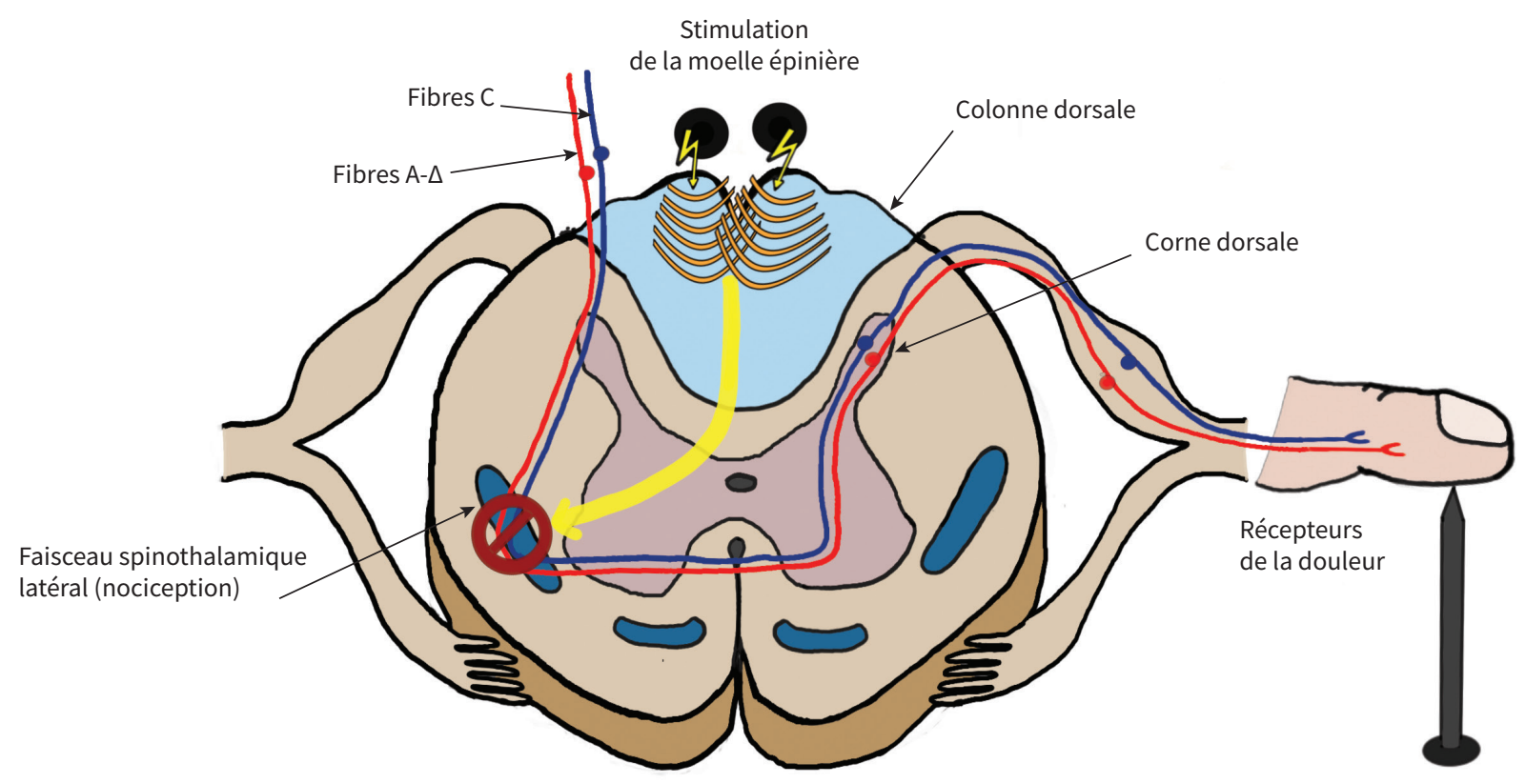

Coupe transversale de la moelle épinière

Figure 1 : Théorie dite du "portillon » appliquée à la douleur. Selon cette théorie, la transmission des signaux douloureux par le faisceau spinothalamique à partir des fibres qui les transmettent (fibres $C$ non myélinisées et fibres $A-\Delta$ légèrement myélinisées) est bloquée par la stimulation des grosses fibres myélinisées de la colonne dorsale et des interneurones polysynaptiques qui assurent la décharge neurale vers la colonne dorsale, bloquant les récepteurs de la douleur au niveau des synapses. Illustration par Jean Chan.

expliquer le soulagement de la douleur. Selon cette théorie, les influx non douloureux referment les "portillons nerveux» pour bloquer l'impulsion douloureuse, l'empêchant ainsi d'atteindre le cerveau ${ }^{2,7}$ (figure 1).

\section{Comment le traitement est-il administré?}

Initialement, les stimulateurs de la moelle épinière étaient implantés par des chirurgiens spécialistes et nécessitaient une anesthésie générale, une laminectomie, la pose d'électrodes directement sur la dure-mère et une hospitalisation postopératoire. Au cours de la dernière décennie, le développement d'électrodes épidurales percutanées insérées sous guidage fluoroscopique a permis à des anesthésiologistes dûment formés ou à d'autres spécialistes de la douleur d'implanter de manière sécuritaire les stimulateurs de la moelle épinière chez les patients en clinique externe, avec une légère sédation et une anesthésie locale (figure 2).

La stimulation de la moelle épinière est administrée en 2 phases : une phase d'essai, suivie, si elle est concluante, de l'implantation permanente.

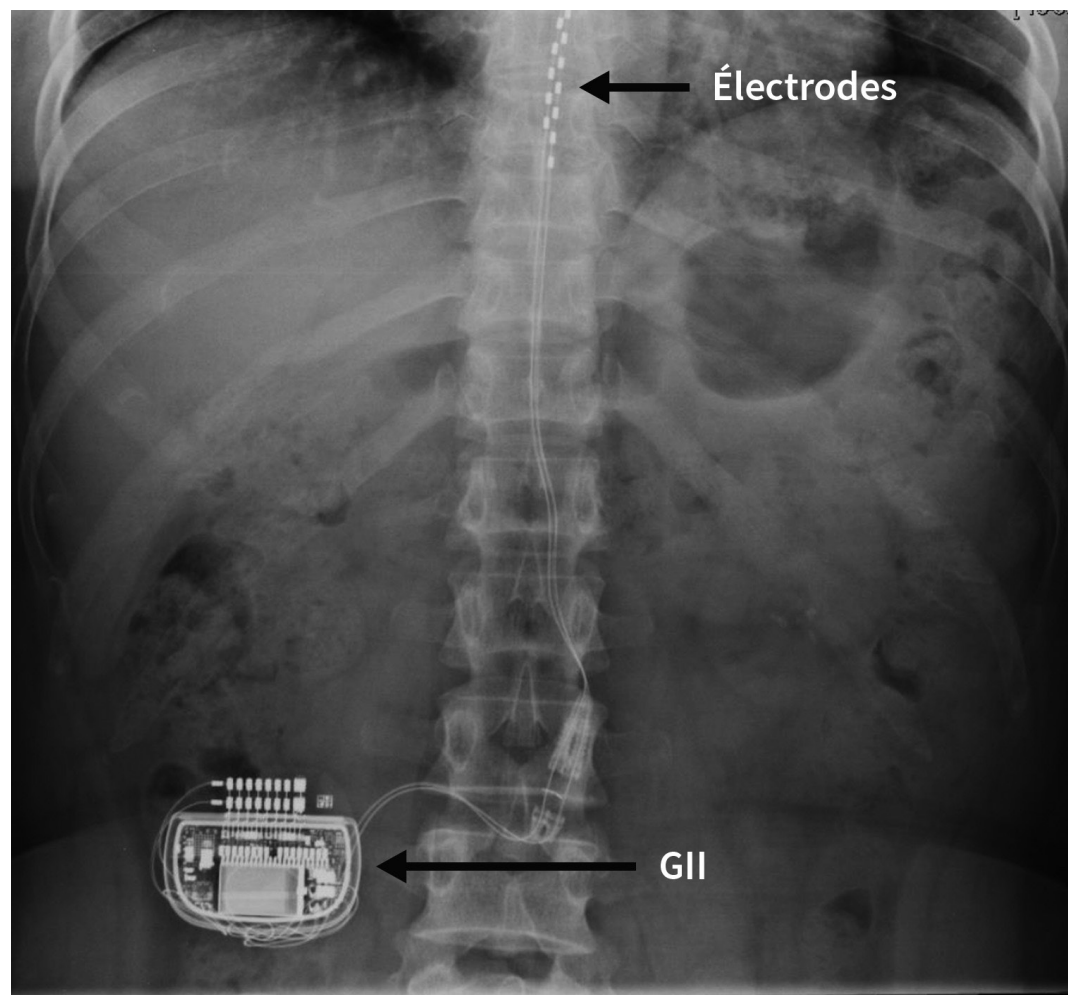

Figure 2 : Radiographie d'un stimulateur de la moelle épinière. Remarque : GII = générateur d'impulsions implantable. 
L'implantation d'un dispositif à l'essai pendant environ 1 semaine permet d'en déterminer l'efficacité2. II faut obtenir une amélioration de $50 \%$ ou plus par rapport à la douleur de départ pour envisager l'implantation d'un dispositif permanent ${ }^{2,7}$.

À cette fin, on peut demander une consultation pour une éventuelle stimulation de la moelle épinière auprès de la plupart des spécialistes de la douleur ou des chirurgiens de la colonne vertébrale des établissements affiliés à des universités canadiennes.

\section{Qui est admissible?}

Selon les rapports, la plupart des maladies neuropathiques chroniques répondent à la stimulation de la moelle épinière, y compris le syndrome d'échec de la chirurgie du dos, le syndrome douloureux régional complexe et les neuropathies périphériques chroniques ${ }^{2,4,8}$. D'autres indications sont en cours d'évaluation, soit l'angine réfractaire, la névralgie diabétique, la névralgie postzostérienne et la douleur ischémique viscérale ou périphérique $e^{2,4}$.

Les contre-indications incluent infections actives, immunosuppression et incapacité de tolérer un traitement anticoagulant ${ }^{2}$. Les personnes qui ont été victimes de violence ou de traumatisme, qui ont un piètre réseau social et qui présentent des déficits cognitifs substantiels ont tendance à obtenir de moins bons résultats et on recommande pour elles une évaluation par des experts en santé mentale ${ }^{2}$.

\section{Quels sont les préjudices potentiels?}

La stimulation de la moelle épinière est généralement sécuritaire parce qu'elle est réversible et minimalement effractive. Les complications les plus fréquentes sont liées au matériel, telles que la migration des électrodes ou leur bris et la défaillance du générateur d'impulsions implantable, mais elles sont facilement remédiables $^{9}$. Au chapitre des complications biologiques, moins fréquentes, mentionnons séromes au point d'insertion du générateur $(2,5 \%)$, infections locales $(3,4 \%)$ et céphalées postponction durale $(0,3 \%)$. Les complications graves comme les abcès ou hématomes épiduraux et les lésions neurologiques sont également rares $(<0,3 \%)^{9}$.

\section{Quelles sont les données probantes?}

Dans une revue systématique de 2020 regroupant 15 essais randomisés et contrôlés, Hofmeister et ses collaborateurs ont recensé un solide corpus de données probantes à l'appui de la stimulation de la moelle épinière ${ }^{4}$. On trouve ci-dessous une description de 3 des plus influentes études réalisées sur l'efficacité du traitement.

L'étude PROCESS (Patients with Failed Back Surgery Syndrome), réalisée en 2007, est un essai international, multicentrique, randomisé, qui a comparé la stimulation tonique de la moelle épinière plus prise en charge médicale, à la prise en charge médicale seule chez 100 patients présentant un syndrome d'échec de la chirurgie du dos. Le paramètre principal était une amélioration de $50 \%$ ou plus de la douleur périphérique sur une échelle analogique visuelle (ÉAV) de $10 \mathrm{~cm}$. Après 12 mois, ce paramètre était atteint par $34 \%$ des patients qui recevaient la stimulation de la moelle épinière, contre $7 \%$ des patients traités médicalement seulement $(p=0,005)$. Pour les paramètres secondaires, les patients qui recevaient la stimulation de la moelle épinière ont présenté une amélioration de leur qualité de vie et de leur capacité fonctionnelle, et une satisfaction plus grande à l'endroit du traitement. En outre, 8 patients sur 50 (16\%) ayant reçu la stimulation de la moelle épinière ont cessé leurs opioïdes, contre 1 patient sur $44(2 \%)$ traité médicalement seulement ${ }^{6}$.

L'étude Senza est un essai de non-infériorité multicentrique, randomisé, pragmatique, avec groupe parallèle, qui comparait la stimulation de la moelle épinière à haute fréquence à la stimulation tonique classique chez 198 patients souffrant de douleur rebelle et chronique au dos et aux jambes. Le paramètre principal était une réduction de $50 \%$ ou plus de la douleur au dos ou aux jambes sur une ÉAV de $10 \mathrm{~cm}$ en l'absence d'un déficit neurologique lié à la stimulation. À 12 mois, la stimulation à haute fréquence s'est révélée supérieure à la stimulation tonique pour ce qui est de réduire la douleur au dos et aux jambes (79\% c. 51,3\%; $p<0,001)$. Les paramètres secondaires incluaient la réduction ou l'élimination de la consommation d'opioïdes, qui était plus marquée dans le groupe soumis à la stimulation de haute fréquence $(35,5 \%)$ que dans le groupe sous stimulation tonique $(26,4 \% ; p<0,05)^{10}$.

L'étude SUNBURST (Success Using Neuromodulation With BURST) comparait la stimulation en rafale à la stimulation tonique chez 100 patients ${ }^{7}$. Cet essai de non-infériorité multicentrique, randomisé et contrôlé avec permutation des groupes a fait état de la supériorité de la stimulation en rafale par rapport à la stimulation tonique chez chaque patient pour ce qui est de l'amélioration globale sur une ÉAV de $100 \mathrm{~mm}$. Durant la phase en rafale, $89 \%$ des patients ont éprouvé une paresthésie réduite voire nulle. Après la période de permutation initiale et 1 an de suivi, $68,2 \%$ ont préféré la stimulation en rafale comparativement à $23,9 \%$ qui ont préféré la stimulation tonique $(p<0,001)^{8}$.

Pour ces essais, on a levé l'insu, rendu impossible en raison de l'expérience de la paresthésie. Au cours des 3-5 dernières années, la stimulation de la moelle épinière est une technologie qui a évolué, avec la mise au point d'une stimulation ne provoquant pas de « paresthésie $»^{2}$. Un stimulus inférieur au seuil de perception est produit par une stimulation de la moelle épinière à haute fréquence et des impulsions de faible amplitude $(30 \mu \mathrm{s} 10 \mathrm{kHz})$ et par une stimulation de la moelle épinière en rafale émulant la décharge neuronale au moyen d'une stimulation en rafale en alternance avec une phase de récupération ${ }^{8}$. La stimulation en rafale et à haute fréquence semble supérieure à la stimulation tonique classique $^{8,10}$. Les dispositifs ne provoquant pas de paresthésie ouvriraient éventuellement la porte à des études à l'insu, et des études à plus long terme permettraient de vérifier l'efficacité de la stimulation de la moelle épinière au-delà de 2 ans d'utilisation.

\section{Rentabilité}

Les traitements de la douleur dorsale et périphérique chronique ont de tout temps représenté un fardeau en raison de leurs coûts initiaux et récurrents élevés. Hoelscher et ses collaborateurs ${ }^{7}$ ont passé en revue les données relatives aux coûts tirées de 21 études 
rétrospectives. Cinq études ont procédé à des analyses coûtefficacité et les résultats se situaient à l'intérieur des seuils habituels de "volonté de payer »des tierces parties, soit entre 50000 \$ et 100000 \$ US par AVAQ (année de vie en fonction de la qualité) gagnée. L'information sur le rapport coût-efficacité à long terme s'est principalement limitée à une modélisation des données relatives aux coûts directs, mais la durée des traitements de stimulation de la moelle épinière donne à penser que les coûts initiaux seraient récupérés en l'espace de 2 ou 3 ans $^{7}$. Il faudra compiler des données prospectives à long terme tirées d'essais randomisés incluant les coûts directs et indirects.

\section{Que nous réserve l'avenir?}

Les dispositifs de neuromodulation récemment approuvés par Santé Canada permettent une stimulation du ganglion spinal plutôt que de la moelle épinière, ce qui requiert moins d'énergie et traite un plus grand nombre de dermatomes ciblés. Certains systèmes ne requièrent plus l'implantation du générateur d'impulsions. D'autres perfectionnements ont aussi été apportés au dosage de la puissance des stimuli, à la combinaison des types de stimuli ou à leur ajustement en fonction de la position du corps (stimulateur de la moelle épinière en circuit fermé).

L'amélioration de la technologie des piles devrait faciliter la miniaturisation des générateurs d'impulsions implantables, conférer aux piles plus de puissance ou éliminer le recours à un chargeur externe. La communication à distance pourrait faciliter la reprogrammation à domicile, les dispositifs portables permettraient un suivi des paramètres, p. ex., la moindre augmentation du taux d'activité résultant d'une réduction de la douleur.
À l'heure actuelle, toutes les provinces remboursent la stimulation de la moelle épinière, mais en général, elles limitent le nombre annuel d'implantations de dispositifs. Les données tirées d'essais randomisés qui évaluent le rapport coût-efficacité et les dispositifs plus récents pourraient en favoriser un usage plus généralisé.

\section{Références}

1. Chronic pain in Canada: laying a foundation for action - a report by the Canadian Pain Task Force, June 2019. Ottawa: Health Canada; 2019.

2. Deer TR, Mekhail N, Provenzano D, et al. Neuromodulation Appropriateness Consensus Committee. The appropriate use of neurostimulation of the spinal cord and peripheral nervous system for the treatment of chronic pain and ischemic diseases: the neuromodulation appropriateness consensus committee. Neuromodulation 2014;17:515-50, discussion 550.

3. Ontario Health (Quality). 10-kHz high-frequency spinal cord stimulation for adults with chronic noncancer pain: a health technology assessment. Ont Health Technol Assess Ser 2020;20:1-109.

4. Hofmeister M, Memedovich A, Brown S, et al. Effectiveness of neurostimulation technologies for the management of chronic pain: a systematic review. Neuromodulation 2020;23:150-7.

5. Shealy CN, Mortimer JT, Reswick JB. Electrical inhibition of pain by stimulation of the dorsal columns: preliminary clinical report. Anesth Analg 1967;46:489-91.

6. Kumar K, Taylor RS, Jacques L, et al. Spinal cord stimulation versus conventional medical management for neuropathic pain: a multicentre randomised controlled trial in patients with failed back surgery syndrome. Pain 2007;132:179-88.

7. Hoelscher C, Riley J, Wu C, et al. Cost-effectiveness data regarding spinal cord stimulation for low back pain. Spine 2017;42(Suppl 14):S72-9.

8. Deer T, Slavin KV, Amirdelfan K, et al. Success Using Neuromodulation With BURST (SUNBURST) study: results from a prospective, randomized controlled trial using a novel burst waveform. Neuromodulation 2018;21:56-66.

9. Deer TR, Mekhail N, Provenzano D, et al. Neuromodulation Appropriateness Consensus Committee. The appropriate use of neurostimulation: avoidance and treatment of complications of neurostimulation therapies for the treatment of chronic pain. Neuromodulation 2014;17:571-97.

10. Kapural L, Cong Y, Doust M, et al. Novel $10-\mathrm{kHz}$ high-frequency therapy is superior to traditional low-frequency spinal cord stimulation for the treatment of chronic back and leg pain (SENZA-RCT). Anesthesiology 2015;123:851-60.
Intérêts concurrents : Aaron Hong signale avoir reçu des honoraires personnels de Stimwave Canada et des subventions des Laboratoires Abbott et de Medtronic, à l'extérieur des travaux soumis. Aucun autre intérêt concurrent n'a été déclaré.

Cet article a été révisé par des pairs.

Affiliations : Département d'anesthésie (Hong, Hare, Mazer), Hôpital St. Michael's; Département d'anesthésiologie et de médecine de la douleur (Hong, Hare, Mazer), Université de Toronto, Toronto, Ont.; Département d'anesthésie (Varshney), Providence Health Care, Vancouver, C.-B.; Département de physiologie (Hare, Mazer), Université de Toronto; Centre de recherche en sciences biomédicales Keenan de l'Institut du savoir Li Ka Shing (Hare, Mazer), Hôpital St. Michael's, Toronto, Ont.

Collaborateurs : Aaron Hong et Vishal Varshney ont contribué à la conception et à la modélisation du travail et ont rédigé l'ébauche du manuscrit. Tous les auteurs ont participé à l'acquisition et à l'interprétation des données. Tous les auteurs ont révisé de façon critique le contenu intellectuel important du manuscrit; ils ont donné leur approbation finale pour la version destinée à être publiée et assument l'entière responsabilité de tous les aspects du travail.
Propriété intellectuelle du contenu : Il s'agit d'un article en libre accès distribué conformément aux modalités de la licence Creative Commons Attribution (CC BY-NC-ND 4.0), qui permet l'utilisation, la diffusion et la reproduction dans tout médium à la condition que la publication originale soit adéquatement citée, que l'utilisation se fasse à des fins non commerciales (c.-à-d., recherche ou éducation) et qu'aucune modification ni adaptation n'y soit apportée. Voir : https://creativecommons.org/ licenses/by-nc-nd/4.0/.

Correspondance : Aaron Hong, Aaron.Hong@unityhealth.to 